\title{
The Convergence of the telematic, computing and information services as a basis for using artificial intelligence to manage complex techno-organizational systems
}

\author{
Alexander Raikov ${ }^{1}$, Arkady Trachuk $^{2}$, Yulia Romanova ${ }^{2}$, Valery Loginova ${ }^{3, *}$, and Vitalia \\ Bortalevich $^{4}$ \\ ${ }^{1}$ RAS, Institute for Control Sciences, 117218 Moscow, Russia \\ ${ }^{2}$ Financial University under the Government of the Russian Federation, Department of Management, \\ 125993 Moscow, Russia \\ ${ }^{3}$ RAS, Institute of Market Problems, 117418 Moscow, Russia \\ ${ }^{4}$ National Institute of Energy Security, 119992 Moscow, Russia
}

\begin{abstract}
The authors analyses the problems of using artificial intelligence to manage complex techno-organizational systems on the basis of the convergence of the telematic, computing and information services in order to manage complex techno-organizational systems in the aerospace industry. This means getting the space objects a higher level of management based on the self-organizing integration principle. Using the artificial intelligence elements allows us to get more optimal and limit values parameters of the ordinal and critical situations in real time. Thus, it helps us to come closer to the limit values parameters of the managed objects due to rising managing and observant possibilities.
\end{abstract}

\section{Introduction}

The main advantage of using the artificial intelligence to manage complex technoorganizational systems on the basis of the convergence of the telematic, computing and information services is having more opportunities to collect, process, keep and distribute information [1]. In other words, this means the ability to adapt to the ordinal and critical situations dynamics $[2,3]$.

In this way such using the artificial intelligence improves the management system by using more simple and faster planning, setting and controlling space objects which form quasi-unified system [4-6].

The realization of the self-organizing integration principle may be applicable to the various kinds of technical problems. For example, it can be used in constructing the space power plants from the little standard modules launched through electromagnetic accelerators.

\footnotetext{
* Corresponding author: loginovalerochka@mail.ru
} 
The description of each type of identifiable electronic control transactions performed on systems in a network (cloud, swarm) of standard modules as elements of a space power plant contains a specific set of attributes for it.

Descriptions of identifiable electronic control transactions (through dedicated clusters of managed space objects) associated with the identification and analysis of atypical situations of the same type can be grouped into information blocks that represent virtual information blocks for the described schemes for implementing self-organizing integration processes.

\section{Problem statement}

Instrumental support of the processes of analyzing the information resources of objects participating in the processes of self-organizing integration, available for monitoring to structures that implement the functions of automated monitoring and control over the effective and safe operation of complex techno-organizational systems in the aerospace industry in the process of self-deployment in the network orbit (clouds, swarms ) of standard modules as elements of space power plants is as follows.

Integration of information systems of various groups of controlled space objects is provided by standardizing the descriptions of the identifiable electronic control transactions made with respect to systems in the network (cloud, swarm) of standard modules as elements of a space power station when space power stations are built in orbit from small standard modules transceivers and microcontrollers) triggered by electromagnetic accelerators that support the modes of self-organization the integration of a swarm of controlled space objects and the support in each of them of a standard protocol.

Functional relations determine the nature of content formation for automating the procedures for constructing both concepts and denotata, where the basic characteristics of monitoring are established to maintain the regimes of self-organizing integration of a swarm of controlled space objects (including the identification of cause-effect relationships between the dynamics of changes in the modes of operation of the system) renewable and dynamically adapted to the individualized profile modes of self-organizing integration of a swarm of controlled space objects ect.

\section{Solution}

On this basis, it is possible to identify the technological activity of controlled space objects creating increased risks, where the behavior of the participants in technological interaction makes it possible to distinguish the organizational strategy of controlled space objects creating increased risks and their characteristics (chronotype, participants, role structure, etc.).

For example, the analysis of technological schemes with the identification of relevant emergency space objects and an assessment of their significance on the basis of statistics of accidents and incidents with different profiles of objective activity in the network (cloud, ro) of standard modules as elements of a space power plant.

The structural relationships determine the identifiable links of operational-regime situations dynamically localized as temporary, resource or any other matrix that formalizes the information portrait of the process of maintaining the regimes of self-organizing integration of a swarm of controlled space objects using structured content blocks of distributed databases consisting of different data, versions or structured options, typical or atypical.

Affiliation ties determine the affiliation of information packets distributed over computational nodes as elements of a dynamic computing cluster, including structured information materials for building stable operational and communication links of controlled 
space objects with a common organizational information platform and a coordinated information exchange management system for managing a complex of modules ("sides"), .

The core of the coordinated information exchange management system should be a converged information and computing platform, which simultaneously forms the driver for the network interaction of other computing nodes as elements of a dynamic computing cluster.

Such a system should combine information, telematic and computing services for the development of information systems for managing a swarm of controlled space objects as elements of a quasi-unified system.

While conducting monitoring on information and technological chains of information management, monitoring, storage and exchange creates a kind of dynamically changing multilayered network of semantics of analyzed links between identified situations in the processes of self-organizing integration.

Each layer of such a network corresponds to a certain class of links mediating the provision of the concentration of all information on the passage of each process of selforganizing integration and all together, on the ongoing transactions and participants in technological interaction for self-deployment of standard modules in the orbit of the network (cloud, swarm) as elements of space power plants.

The possibilities of network-centric integration of data with respect to the content of distributed databases, consisting of data received from space and ground objects, provide communication between the monitoring and control operators during the implementation of the whole range of different technological processes for self-deployment in the orbit of the network (clouds, swarms) modules as elements of space power stations.

The detection of the described system-dynamic communication serves as a message to the monitoring and control operator about a new connection within the analyzed distributed databases and test results with the formation of a temporary, resource or operational-regime matrix of the process of self-organizing integration that are carriers of information determined by the class and description of this connection .

The possibility of forming a temporary, resource or operational-regime matrix of the studied process of self-organizing integration is created, which can be based both on a separate event (the result of a technological operation) and on aggregation and comparison of dissimilar technological events and network flows of such operations.

In this complex, a unique opportunity is created for a principled increase in the effectiveness of the organizational mechanisms for reducing the risks of accidents and improving the sustainability of the control processes for the swarm of controlled space objects in direct interaction between the board and the aircraft, or when transmitting on board each module information from the ground-based surveillance system.

The complex makes it possible to detect the interrelationships between controlled space objects, actors, events and various implicit correlations between them with respect to the detection, based on analysis of monitoring results, of the explicit and latent characteristics of the process of providing self-organizing integration (formation of the core of an intelligent generator and energy translator, integrable from separate quasi-autonomous elements in a network (cloud, swarm) of standard modules) and its correspondences normative requirements.

As a result, there is a distributed-network formation of a set of measures to prevent emergencies, situations with non-fulfillment or inadequate execution of regulations while maintaining the regimes of self-organizing integration of a swarm of controlled space objects with a constant step-by-step comparison with the required normative model of using complex techno-organizational systems in the aerospace industry for self-deployment at Orbit network (cloud, swarm) of standard modules as elements of space power plants. 
To ensure the safety and effective use of complex techno-organizational systems in the aerospace industry in the process of self-organizing integration, a method of intellectual analysis of the fractal organization of key element relationships is proposed, using a multidimensional interpretation of the linkage systematics and operational-regime control transactions.

The new technology provides a dynamic study of the semantics of the analysis of explicit and latent relationships in databases containing the necessary information about operational-regime situations.

\section{Conclusion}

Automated monitoring and control is performed to determine the basic characteristics of the system-dynamic analysis of the electronic content of control transactions while maintaining the regimes of self-organizing integration of a swarm of controlled space objects, testing the time, resource or operational-mode matrix of any studied self-organizing integration process energy from various points of geostationary orbits of space solar power stations on the ground.

The quasi-unified system of space object management has some principal differences from the traditional one and that makes it substantially new one.

In order to improve the functioning of the system it is necessary to add the next elements to the using artificial intelligence process:

1) The monitoring subsystem of the measurable parameters of the various kinds of physical fields, environments and objects which provide the forming of the current space objects modules situation.

2) The diagnostics of anomalies subsystem that will identify the anomaly`s reasons and determine the current situation on controlled objects.

3) The analysis of the current indicators subsystem which will evaluate the functionality of stabilization process of the space objects (including adding and reducing of the elements)

4) The subsystem of evaluation of the current plan of the telematic, computing and information services management which analyzes the working system and warns if there are problems.

5) The intellectual agent which is planning and correcting the work of the telematic, computing and information services.

Application part should include the following modules:

- the real-time monitoring of elements and processes;

- the modeling, recognizing and analyzing system of the global and current situation and supporting decision-making and in the ordinary conditions;

- the system for predicting problems in real time;

- the intelligent real-time agent for correcting the management and optimizing solutions;

- the interface for making recommendations to the modules in a critical situation.

The theses were prepared with the financial support of the Russian Humanitarian Scientific Foundation (project No. 16-02-00463 a "Formation of organizational mechanisms for operating oil and gas resources on the basis of multi-agent modeling to protect Russia's economic interests from manipulating oil prices on world markets").

\section{References:}

1. A. S. Bugaev, E. L. Loginov, A. N. Raikov, V. N. Saraev, S\&T. Inf. Pr. 36 (1), 68 (2009)

2. E. L. Loginov, A. N. Raikov, Therm. Eng. 62(4), 233 (2015) 
3. A. P. Grigorev, A. I. Soldatov, P. V. Sorokin, MTT, 97 (2000)

4. D. N. Demyanovich, O. S. Vadutov, A. I. Soldatov, MEACS, (2014)

5. A. S. Asochakov, Y. V. Shulgina, A.I . Soldatov, E. M. Shulgin, D. N. Ogorodnikov, MEACS, (2015)

6. S. V. Chubov, A. I. Soldatov, IOP Conf. Ser. 177(1), (2016) 